\title{
Multivariate risk prediction tools including MRI for individualized biopsy decision in prostate cancer diagnosis: current status and future directions
}

\author{
Ivo G. Schoots ${ }^{1}$ (I) Monique J. Roobol ${ }^{2}$
}

Received: 19 December 2018 / Accepted: 25 February 2019 / Published online: 13 March 2019

(c) The Author(s) 2019

\begin{abstract}
Background and purpose Individualized risk-adapted algorithms in prostate cancer (PCa) diagnosis using predictive prebiopsy variables in addition to prostate-specific antigen value may result in a considerable reduction of unnecessary systematic biopsies. Multi-parametric magnetic resonance imaging (mpMRI) has emerged as a secondary prediction tool that can further improve the detection of clinically significant prostate cancer (csPCa). This review explores the performance of new MRI risk models for indicating a biopsy for prostate cancer diagnosis.

Results and considerations The area under the receiver-operating characteristic curve for detecting csPCa varies between 0.64 and 0.91 in biopsy-naïve men, and between 0.78 and 0.93 in men with a previous negative biopsy. The utility of multivariate risk prediction tools including MRI suspicion scores as an extra input parameter has the potential to avoid a notable number of biopsies and detection of clinically insignificant $\mathrm{PCa}$ at a low price of missing some csPCa. The trade-off depends on the risk threshold that is chosen. In biopsy-naïve men a net benefit was obtained at a risk threshold of above $10 \%$ for csPCa in most MRI risk prediction models. All constructed MRI risk models used (referral) patient cohorts with high prevalence of csPCa. Using more representative cohorts from daily clinical screening, net benefit may attenuate at lower risk thresholds. Strengths and limitations of these models are discussed.

Future directions To assess their wider applicability, in-depth analysis of mpMRI predictive qualities should be further investigated, in combination with required external validation of these models in a multicenter setting with large prospective datasets.
\end{abstract}

Keywords Prostate cancer (PCa) · Biopsy $\cdot$ Magnetic resonance imaging (MRI) $\cdot$ MRI-guided targeted biopsy $\cdot$ PI-RADS · Risk stratification

\section{Introduction}

Transrectal ultrasound-guided systematic biopsy in the work-up of prostate cancer (PCa) diagnosis has shown a rising prevalence of antibiotic-resistant bacterial infections with biopsy-related septic complications [1]. Furthermore, systematic biopsy is associated with increased detection

Ivo G. Schoots

i.schoots@erasmusmc.nl

1 Department of Radiology and Nuclear Medicine, Erasmus MC University Medical Center, P.O. Box 2040, 's-Gravendijkwal 230, 3000 CA Rotterdam, The Netherlands

2 Department of Urology, Erasmus MC University Medical Center, Rotterdam, The Netherlands of indolent or low-grade PCa [2]. Reduction of systematic biopsies is pivotal in men who eventually prove to have no or low-grade PCa. Utility of validated multivariate risk prediction models to determine the risk of clinically significant $\mathrm{PCa}(\mathrm{csPCa})$ and to reduce (unnecessary) biopsy is nowadays recommended in guidelines [3].

The individualized risk-adapted approach in prostate cancer diagnosis is about to change with the introduction of prostate multi-parametric magnetic resonance imaging (mpMRI) in daily practice. Despite the qualities of mpMRI in predicting the absence or presence of csPCa, today mpMRI is still utilized as a diagnostic test for improving the performance of the diagnostic work-up, and not reducing biopsy [3, 4]. In other words, mpMRI is indicated when systematic biopsy is indicated, and thus when the likelihood 
is high of having clinically significant disease in a subsequently systematic biopsy.

mpMRI is especially indicated in men with prior negative biopsy who are still suspected of having significant disease. However, in biopsy-naïve men mpMRI is also suggested as an upfront or prebiopsy diagnostic test, to improve the diagnostic yield when combining targeted and systematic biopsy [5-7]. Moreover, mpMRI has also been introduced as a triage test to indicate performing or not performing a biopsy [5, 8-10]. As a result of its high negative predictive value, men with no suspected evidence for csPCa on MRI may defer systematic biopsy $[11,12]$.

Utilizing mpMRI as a triage test shows resemblance with risk prediction, and may have overlap with current multivariate risk prediction models for prostate cancer [13-19]. Most of these current risk models have been externally validated several times, and all use prostate-specific antigen (PSA), and digital rectal examination (DRE) as individual predictive input variables. To improve their predictive value, some use extra input variables such as age, prostate volume, free PSA, family history, race, and prior negative biopsy.

Due to the predictive value of mpMRI in PCa diagnosis, recently new multivariate risk prediction tools have been constructed, with the inclusion of the mpMRI suspicion score [20-29]. The purpose of this review is to explore the performance of these new MRI risk models for indicating a biopsy for prostate cancer diagnosis.

\section{Multivariate risk prediction including mpMRI for indicating targeted biopsy}

Including the mpMRI as an extra diagnostic test in multivariate risk prediction tools, the balance between benefit and risks may change and should be (re-)evaluated. In this evolution, the first studies are published, mainly focusing on maximizing diagnostic yield, in combinations with potentially reducing biopsies and reducing the detection of clinically insignificant PCa. Study characteristics are shown in Tables 1 and 2. Theses multivariate prediction tools are based on logistic regression models. For these models, mostly the area under the receiver-operatingcharacteristic (ROC) curve (AUC) are investigated for any $\mathrm{PCa}$ and csPCa (Table 2), sometimes in combination with decision and net reduction curve analysis. In most studies clinically significant $\mathrm{PCa}$ was defined as Gleason score $3+4$ or higher, nowadays better referred to the International Society of Urological Pathology (ISUP) grade (G) 2 and higher.

\section{In biopsy-naïve setting}

Several studies have developed an MRI risk prediction model, which some have been internally or externally validated.

Based on the ERSPC-RCs, a risk calculator including mpMRI was developed on datasets from five international centers [20]. Input variables were PSA, DRE, prostate volume, prior biopsies, age, and mpMRI (PI-RADS 1-5) (Table 2). In total, data from 504 biopsy-naïve men were used to adjust the ERSPC-RC3 into the MRI-ERSPCRC3. MRI-ERSPC-RC3 had a significantly higher AUC for $G \geq 2 \mathrm{PCa}$ compared with the non-calibrated baseline model (ERSPC-RC3 + DRE): 0.84 [95\% confidence interval (CI) $0.81-0.88]$ versus 0.76 [0.71-0.80] $(p<0.01)$ (Table 2). In decision curve analysis, the MRI-ERSPC-RC3 showed a benefit for $\mathrm{G} \geq 2 \mathrm{PCa}$ threshold probabilities larger than $10 \%$. For example, at a risk threshold of $\geq 10 \% G \geq 2 \mathrm{PCa}$ to indicate a biopsy, $14 \%(143 / 1000)$ biopsies would have been avoided, missing $G=1$ PCa in 13\% (18/143) and missing $G \geq 2$ PCa in $10 \%$ (14/143) of the avoided biopsies (i.e., negative test) (Table 3 ). We need to address that the prevalence of $42 \% G \geq 2 \mathrm{PCa}$ in this cohort was rather high. At this threshold, still only $3.3 \%$ of all $G \geq 2 \mathrm{PCa}(14 / 423)$ was not detected overall. Due to this high-risk population, hardly any benefit was reached at a risk threshold of lower than $10 \% G \geq 2$ PCa.

Radtke and coworkers [21] investigated multivariate prediction modeling in a similar approach, also based on the ERSPC-RC3. A single-center dataset of 660 biopsy-naïve men was used. MRI-ERSPC-RC3 reached a higher AUC (0.83), compared with ERSPC-RC3 (0.81), age refitted ERSPC-RC3 $(0.80, p<0.001)$, and PI-RADSv1.0 (0.76, $p<0.001$ ) (Table 2). In decision curve analysis, the MRIERSPC-RC3 showed a benefit for $G \geq 2$ PCa threshold probabilities larger than $10 \%$ (Table 3). Also in this study cohort, the estimated prevalence for $G \geq 2 \mathrm{PCa}$ was $46 \%$ and was rather high, explaining the benefit in only the risk threshold above $10 \%$.

Mehralivand and colleagues developed a risk prediction model, based on PSA, DRE, age, African American ethnicity, and incorporating mpMRI (PI-RADS 1-5) [22]. A developing cohort from 1 institute $(n=400)$ and a validating cohort from 2 other institutes $(n=250)$ had prevalence for $G \geq 2 \mathrm{PCa}$ (outcome measurement) of $48.3 \%$ and $38.2 \%$, respectively. In comparison to the baseline model, the AUC of the MRI risk prediction model increased from 0.72 to 0.84 $(p<0.001)$ in the development cohort, and increased from 0.64 to $0.84(p<0.001)$ in the validation cohort (Table 2$)$. By applying the MRI risk prediction model to the validation cohort, higher net benefit than the baseline model could be achieved for risk thresholds above $10 \%$. At a risk threshold 


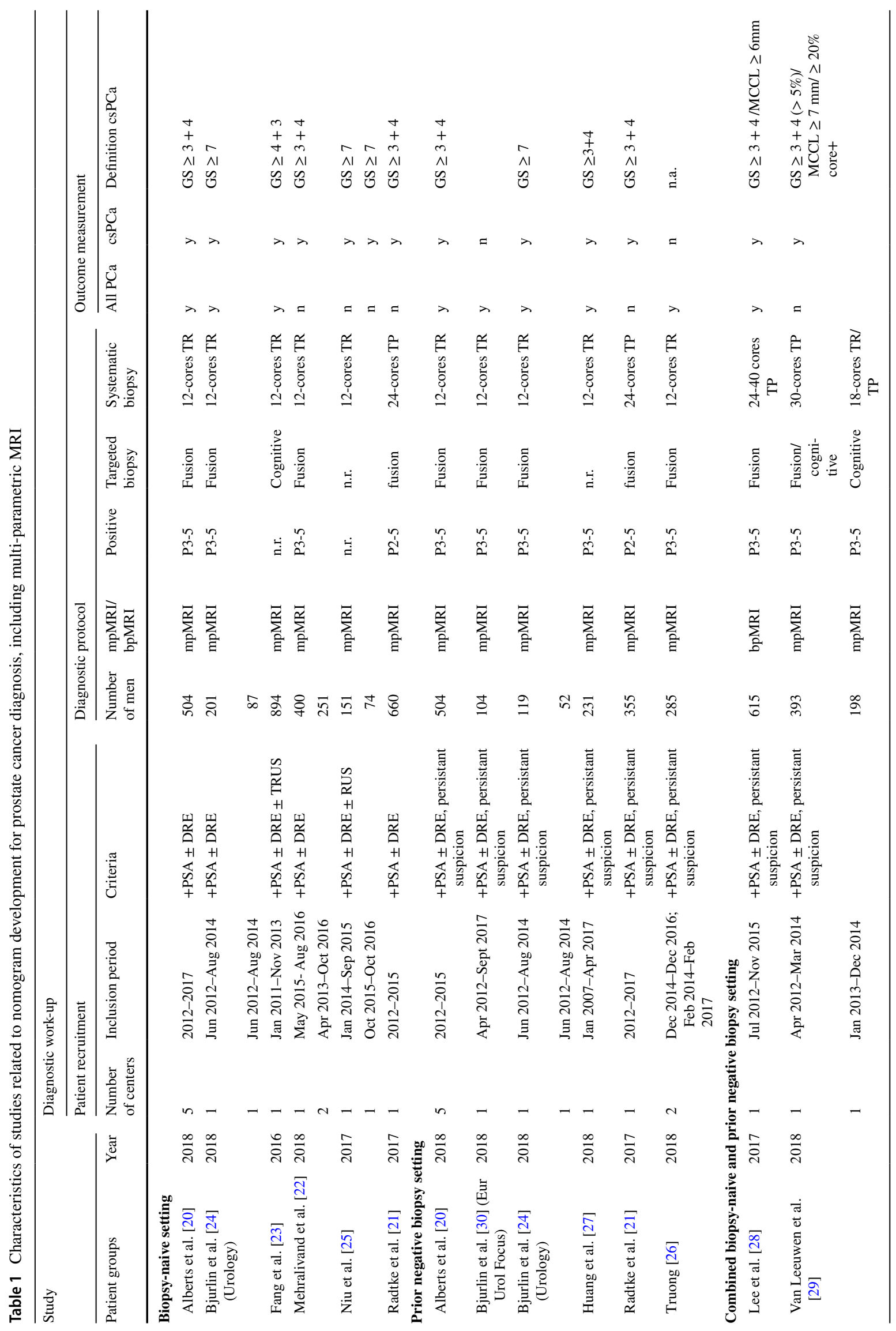


of $\geq 10 \% G \geq 2$ PCa to indicate a biopsy, 17\% (172/1000) biopsies would have been avoided, missing $G \geq 2 \mathrm{PCa}$ in $6 \%(11 / 172)$ in the avoided biopsies (Table 3$)$. In this study cohort, the prevalence for $G \geq 2 \mathrm{PCa}$ was $38 \%$ and was a little lower than in the previous studies, which may contribute to the higher net benefit at the same risk threshold of $\geq 10 \%$.

Fang et al. [23] developed an MRI risk prediction model, based on PSA, age, and (abnormal) transrectal ultrasound, incorporating mpMRI (PI-RADS 1-5). The AUC for $G \geq 3$ PCa for the developing cohort $(n=894$, with a prevalence $24.4 \%)$ was 0.87 , in comparison to $0.85(p=0.001)$ risk prediction model without mpMRI (Table 2). At a risk threshold of $\geq 10 \% G \geq 3$ PCa to indicate a biopsy, $10 \%$ (98/1000) biopsies would have been avoided, missing no $G \geq 3 \mathrm{PCa}$ $(0 \%, 0 / 98)$ in the avoided biopsies (Table 3$)$.

Bjurlin and colleagues [24] developed an MRI risk prediction model, based on PSA-density and age, incorporating mpMRI (MRI suspicion score assessment category 3-5, excluding the first categories). Bias-corrected AUC for $G \geq 2$ (Gleason $\geq 7$ ) PCa was 0.91 for the developing cohort $(n=201$, with a prevalence $34.8 \%)$ and 0.84 for the validating cohort ( $n=87$, with a prevalence $31.0 \%$ ) (Table 2). These AUCs were higher in comparison to baseline model (PSA-density) (0.75 and 0.69) and MRI suspicion score model (0.90 and 0.84).

Niu and coworkers [25] developed an MRI risk prediction model, based on adjusted PSA-density and age, incorporating mpMRI (PI-RADS 1-5). The AUC for $G \geq 2$ (Gleason score $\geq 7$ ) for the developing and validating cohort was 0.85 [0.79-0.90] $(n=151$, with a prevalence $21.2 \%)$ and 0.82 [0.76-0.89] ( $n=74$, with a prevalence $24.3 \%$ ) (Table 2). These AUCs were higher in comparison to the baseline model (PSA-density) (0.74 [0.66-0.79], $p=0.013)$ and PIRADSv2 score (0.76 [0.71-0.84], $p=0.018)$.

\section{In prior negative biopsy setting}

Next to the development of MRI-ERSPC-RC3 on biopsynaïve men, a risk calculator MRI-ERSPC-RC4 was developed for men with prior negative biopsy $(n=457$, prevalence of $29 \% G \geq 2$ PCa) [20]. MRI-ERSPC-RC4 had a significantly higher AUC for $G \geq 2$ PCa compared with the ERSPC-RC4 + DRE (baseline model): 0.85 (95\% CI $0.81-0.89$ ) versus 0.74 (95\% CI $0.69-0.79, p<0.01$ ) (Table 2). Using a $\geq 10 \%$ risk threshold for $G \geq 2$ PCa to indicate a biopsy, 36\% (361/1000) biopsies would have been avoided, missing $G=1 \mathrm{PCa}$ in $15 \%(55 / 361)$ and missing $G \geq 2$ PCa in $4 \%(15 / 361)$ of all avoided biopsies (Table 3 ). In this cohort the prevalence of $29 \% G \geq 2$ PCa was lower than for the biopsy-naïve cohort. Therefore, in contrast to biopsy-naïve men, the decision curve analysis in men with prior negative biopsy already showed clear net benefit of the MRI-ERSPC-RC4 at a risk threshold of $\geq 5 \%$ for $G \geq 2$ PCa (Table 3).

Radtke and coworkers [21] also developed an MRIERSPC-RC4 for men with prior negative biopsy, next to an MRI-ERSPC-RC3. In men with previous biopsy $(n=355$, estimated prevalence of $40 \% G \geq 2 \mathrm{PCa}$ ), the discrimination of the MRI-ERSPC-RC4 (0.81) was superior to that of ERSPC-RC4 (baseline model) $(0.66, p<0.001)$, refitted ERSPC-RC4 (0.76, $p<0.001)$, and PI-RADSv1.0 (0.78, $p<0.001$ ) (Table 2). In decision curve analysis, the MRIERSPC-RC4 showed a benefit for $G \geq 2$ PCa threshold probabilities larger than $\geq 10 \%$ (Table 3 ).

Bjurlin and colleagues [24] investigated an MRI risk prediction model with a bias-corrected AUC for $G \geq 2 \mathrm{PCa}$ of 0.86 for the developing cohort $(n=119$, with a prevalence $21.8 \% G \geq 2 \mathrm{PCa}$ ) and 0.87 for the validating cohort $(n=52$, with a prevalence $9.6 \% G \geq 2 \mathrm{PCa}$ ) (Table 2). These AUCs were higher in comparison to the baseline model (PSAdensity) (0.76 and 0.76 ), and MRI suspicion score model (0.83 and 0.84).

Truong et al. [26] developed an MRI risk prediction model, based on age, PSA, and prostate volume, incorporating mpMRI (PI-RADS 1-5). The AUC for predicting benign prostate pathology for the developing cohort $(n=285$, with a prevalence $46.3 \%$ benign prostate) was 0.83 (Table 2 ). With a cutoff probability of $\geq 0.70$ used to recommend deferment of MRI-targeted biopsy, in 21.4\% (61/285) men unnecessary biopsy would have been avoided, and $6.2 \%(4 / 61)$ with a $G \geq 2$ PCa would have been missed. The prevalence of $G \geq 2$ $\mathrm{PCa}$ in this cohort was 39\% (111/285). Overall, only $3.6 \%$ (4/111) of all $G \geq 2$ PCa would not have been detected at this threshold. Another group [30] validated this prediction tool, and found for predicting benign prostate pathology an AUC of 0.78. An updated model was constructed with improved calibration and similar discrimination (AUC 0.79).

Huang et al. [27] developed an MRI risk prediction model, based on age, PSA, prostate volume, and DRE, incorporating mpMRI (PI-RADS 1-5). The AUC for $G \geq 2 \mathrm{PCa}$ for the developing cohort $(n=231$, with a prevalence $25.5 \%)$ was $0.93[0.89-0.96](p<0.001)$ (Table 2).

\section{In combined biopsy-naïve and prior negative biopsy setting}

Lee et al. [28] developed an MRI risk prediction model, based on age, PSA-density, and primary biopsy, incorporating biparametric MRI (PI-RADS 1-5). The AUC for csPCa ( $G \geq 2$ or MCCL $\geq 6 \mathrm{~mm}$ ) for the developing cohort ( $n=615$, with a prevalence $38.5 \%$ ) was 0.92 [0.89-0.94] (Table 2). At a calculated probability cutoff of $\geq 10 \%$ csPCa to indicate a biopsy, 10.6\% (65/615) biopsies would have been avoided, missing $G=1 \mathrm{PCa}$ in $16.9 \%$ (11/65) and missing $\mathrm{G} \geq 2 \mathrm{PCa}$ in $3.1 \%(2 / 65)$ of the avoided biopsies (Table 3$)$. 


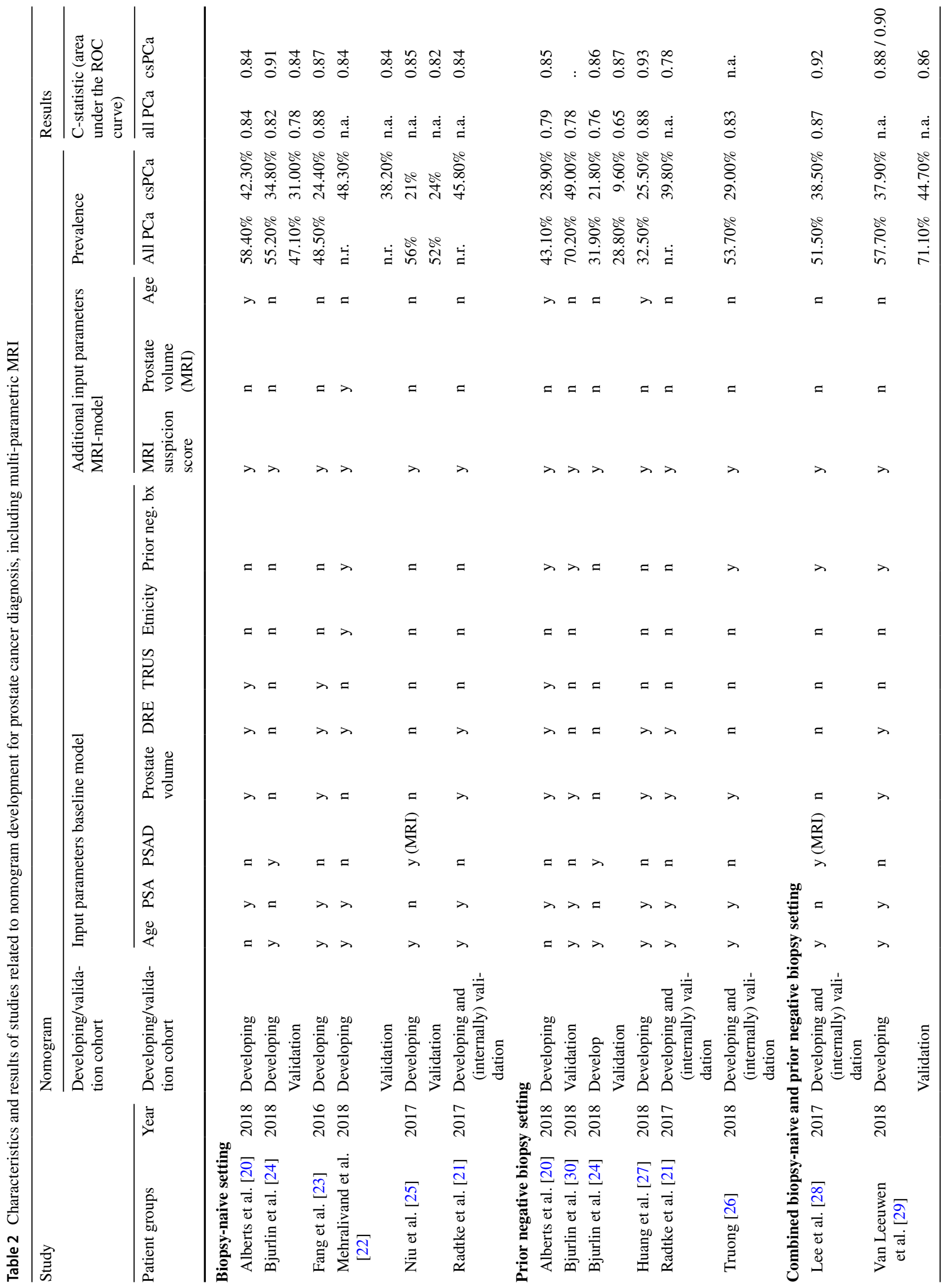




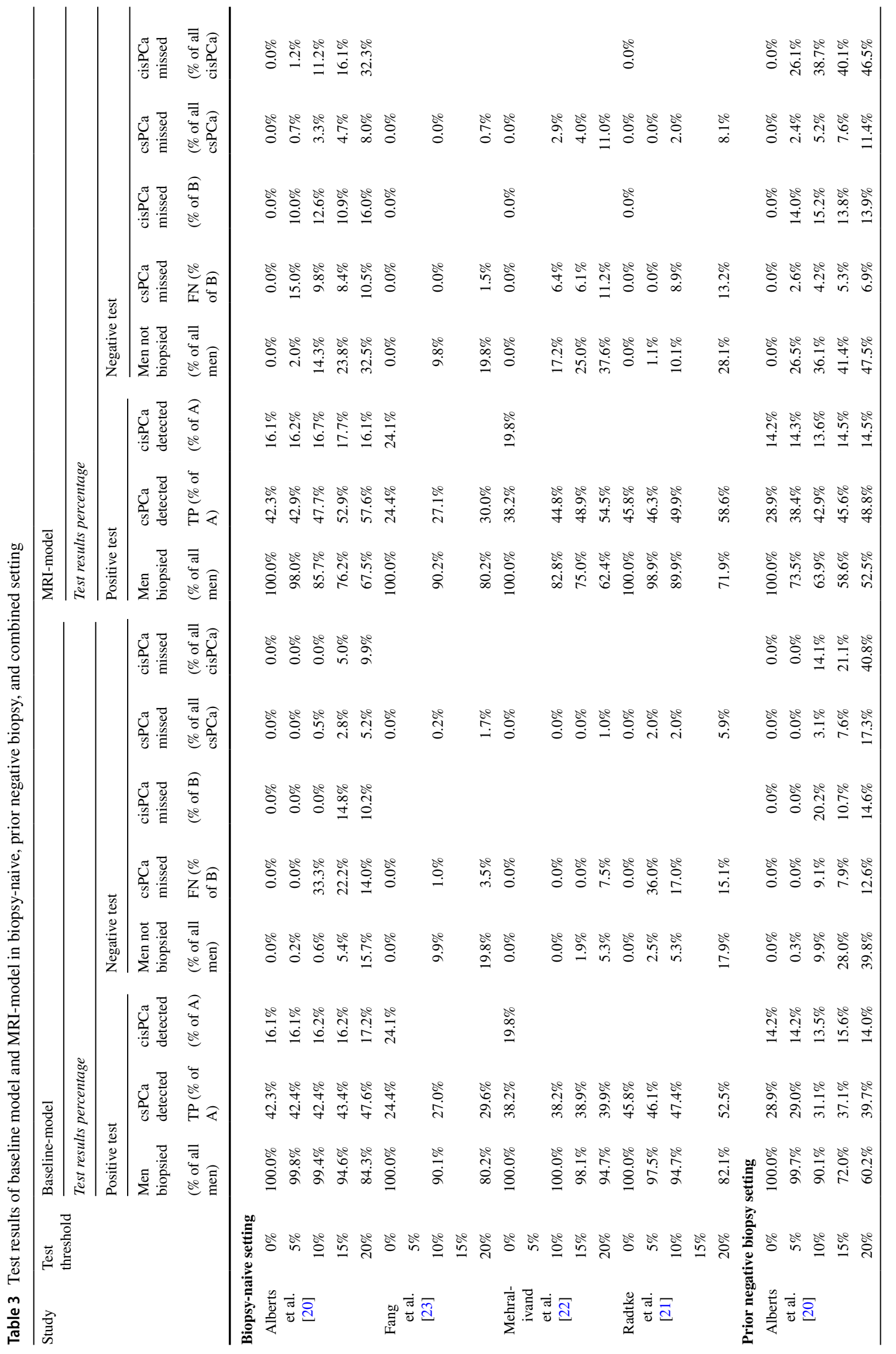




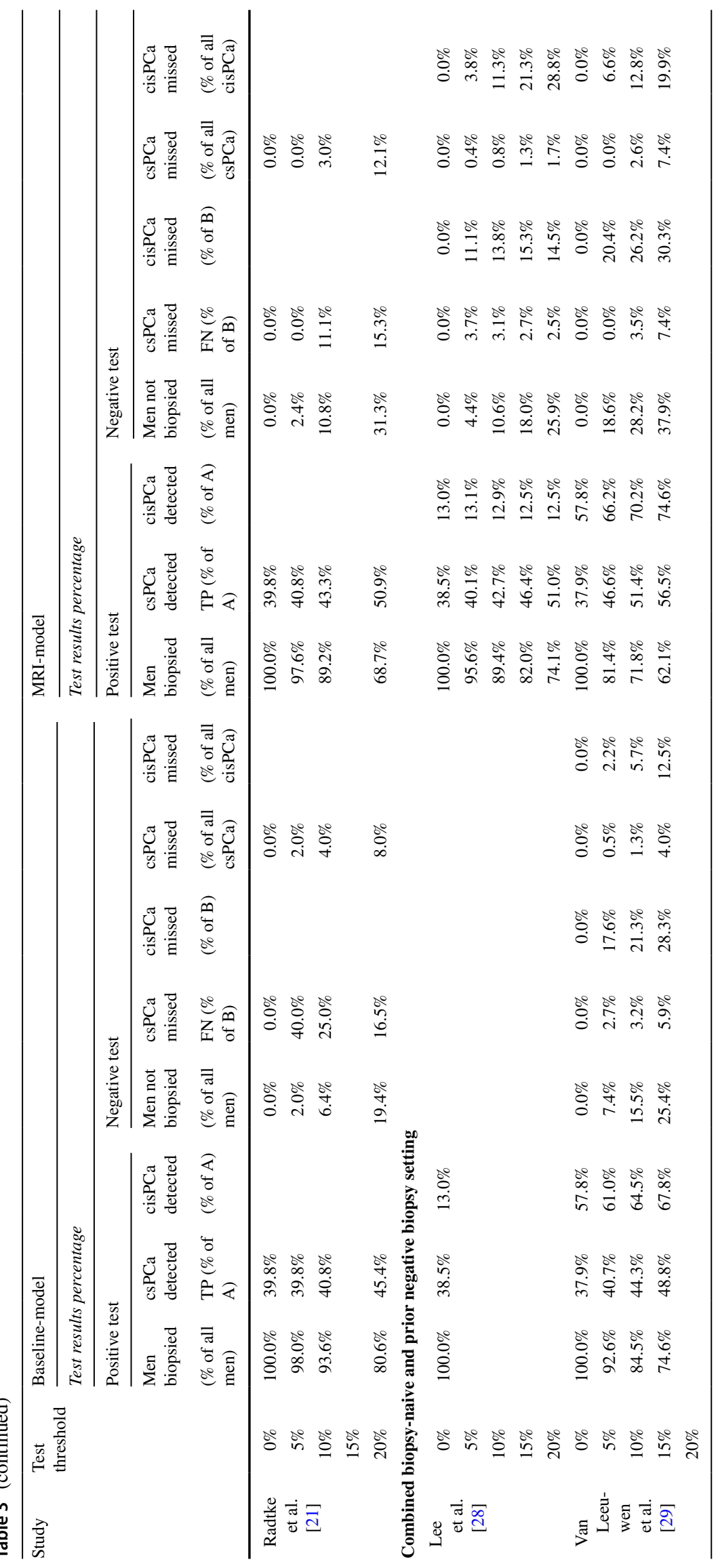


Van Leeuwen and coworkers [29] developed a risk prediction model, based on age, PSA, DRE, prostate volume, previous biopsy, incorporating mpMRI (MRI suspicion score category 1-5). csPCa was defined as Gleason 7 with $>5 \%$ grade $4, \geq 20 \%$ cores positive or $\geq 7 \mathrm{~mm}$ maximum cancer core length (MCCL). The AUC for csPCa was 0.88 [0.85-0.92] for the developing cohort $(n=393$, with a prevalence $37.9 \%$ ) and 0.86 [0.81-0.92] for the validating cohort ( $n=198$, with a prevalence $44.7 \%$ ) (Table 2). This was higher than in comparison to the multivariate risk baseline model $(0.80$ [0.75-0.84]). With a cutoff probability of $\geq 0.10$ used to indicate a biopsy, in $28.2 \%$ (282/1000) men an unnecessary biopsy would have been avoided, missing $G=1 \mathrm{PCa}$ in $26.2 \%$ (74/282) and missing $G \geq 2 \mathrm{PCa}$ in $3.5 \%$ $(10 / 282)$ in the avoided biopsies. Overall, $12.8 \%$ (74/578) cisPCa and $2.6 \%$ (10/379) csPCa were not detected at this threshold.

\section{Performance analysis of the MRI risk prediction models}

Next to the comparison of AUCs between MRI-models, the performance can also be further explored using other performance parameters (Table 4). For such an analysis, performance parameters like true positive rate (TPR), false positive rate (FPR), net benefit (NB), percentage avoided biopsies (PAB), and percentage avoided clinically insignificant PCa could be investigated, as shown by Mehralivand and coworkers [22]. We were able to calculate these performance parameters for most studies, with risk thresholds ranging from 0 to $20 \%$ (Table 4).

By analyzing the individual performance parameters, physician and patient may be better informed to decide whether to undergo further biopsy testing or no further biopsy testing. A better trade-off can be made, based on the subjective weight of each individual performance parameter. If a patient focuses on maximizing diagnostic yield, the TPR is of vital importance in his decision. If a patient focuses on not to be (unnecessarily) biopsied, the FPR and PAB become more prominent. The net benefit is calculated by the formula $=(\mathrm{TP}-w \mathrm{FP}) / N)$, where $\mathrm{TP}$ is the number of true positive decisions, FP the number of false positive decisions, $N$ is the total number of patients and $w$ is a weight equal to the odds of the risk threshold or cutoff in percentages $(\mathrm{pt} /(1-\mathrm{pt})[31]$.

In most studies, the MRI risk prediction models were compared to baseline risk prediction models (without MRI). The input parameters in the used baseline models differed between studies, as previously discussed. The comparison between the each MRI- and baseline model is in fact the calculated difference between the values of the individual performance parameter (Table 4). For example, using the
MRI-model instead of the baseline model in biopsy-naïve men at a risk threshold of $\geq 10 \%$, Alberts and coworkers showed a net difference of $13.7 \%$ in avoiding biopsies, $11.2 \%$ in avoiding the detection of clinically insignificant PCa, $21.7 \%$ reduction of false positive tests, at the expense of $2.8 \%$ not detected csPCa.

Using the MRI-risk model instead of the baseline risk model in biopsy-naïve men, we observe that three studies show a notable beneficial net difference above the threshold of $>10 \%$, at least for the FPR, NB, avoided biopsies and avoided detection of clinically insignificant PCa [20-22]. These results are at a low price of missing csPCa, even in the investigated cohorts with remarkable high prevalence of clinically significant PCa (42.3-48.3\%) [20-22] (Table 2).

These results would have been even better in more reallife opportunistic screening cohorts, instead of the highly selected cohorts in these tertiary hospitals. Following upfront risk prediction in biopsy-naïve men, the prevalence of csPCa might be in the range of approximately $20-30 \%$ $[13,32]$. Before using such a test recalibration of these multivariate risk prediction tools to the in-hospital diagnosed population is mandatory.

\section{Considerations and future perspectives}

Individualized risk assessment of csPCa using a predictive model that incorporates the mpMRI suspicion score in combination with clinical and biochemical data, allows a considerable reduction in unnecessary biopsies and reduction of the risk of over-detection of clinically insignificant PCa, albeit at the expense of a low number of (not diagnosed) csPCa. Whether or not this has detrimental effect on future metastatic and mortality rates remains to be seen. The MRI risk prediction models highlight their accuracy and power, and suggest that the usage of these tools would allow the identification of patients with significant disease. However, at present external validation and results of calibrating steps are lacking.

We need to acknowledge that previously developed and already validated risk calculators, without mpMRI as an input variable, perform sufficient, but can be further improved by the input of other "biomarkers" such as mpMRI. Despite these satisfactory predictive results and potential improvements in outcomes, MRI risk prediction models may also avoid limitations of these models. For example, prostate volume is an important input variable as it is related to PSA value. Prostate volume is commonly determined on TRUS or in certain circumstances estimated by DRE. The use of a TRUS-based input variable, including the variable of abnormal or suspicious TRUS, is impractical since men who undergo TRUS are also likely to undergo TRUS-guided biopsy. With mpMRI, prostate volume can 


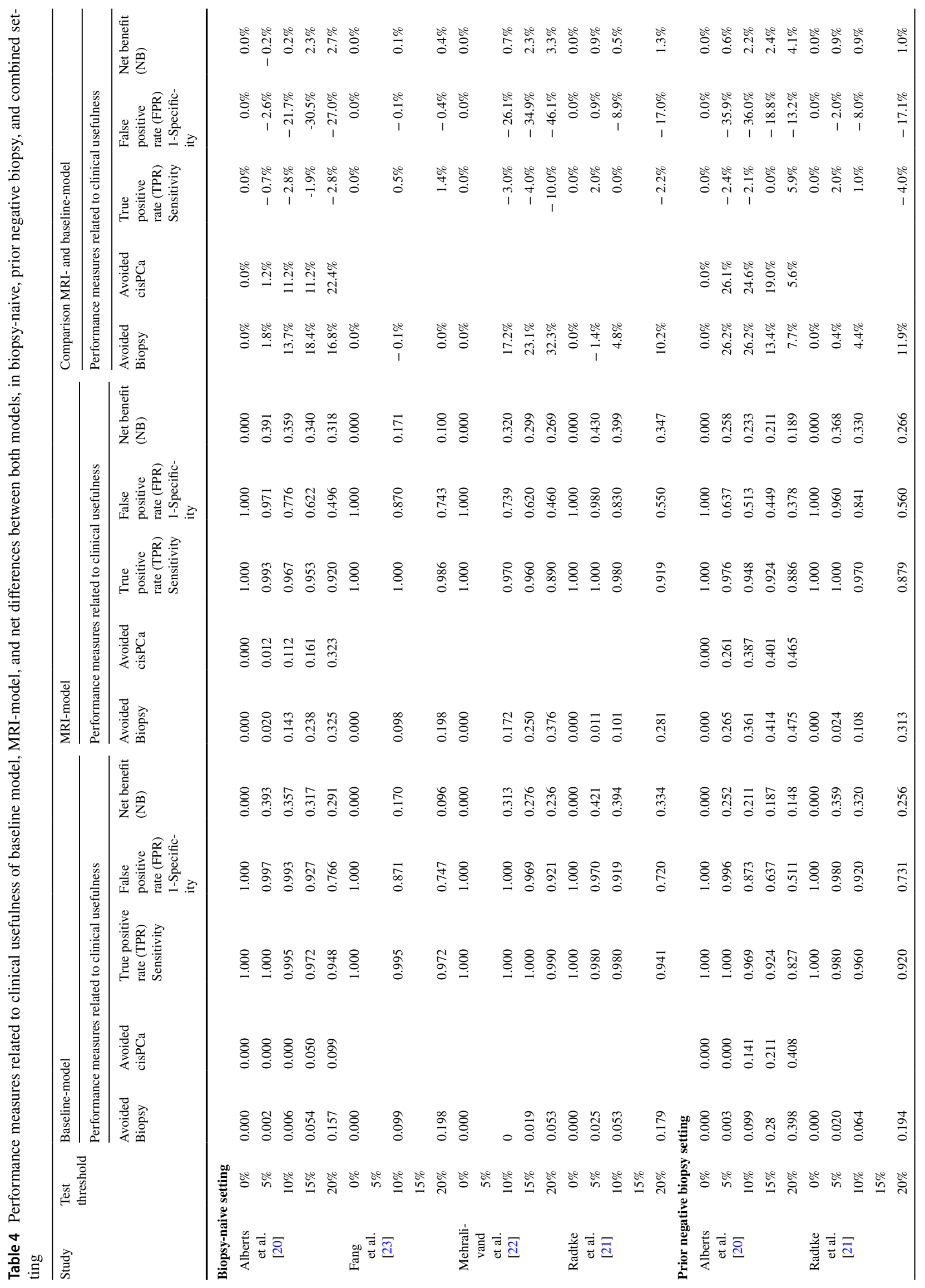




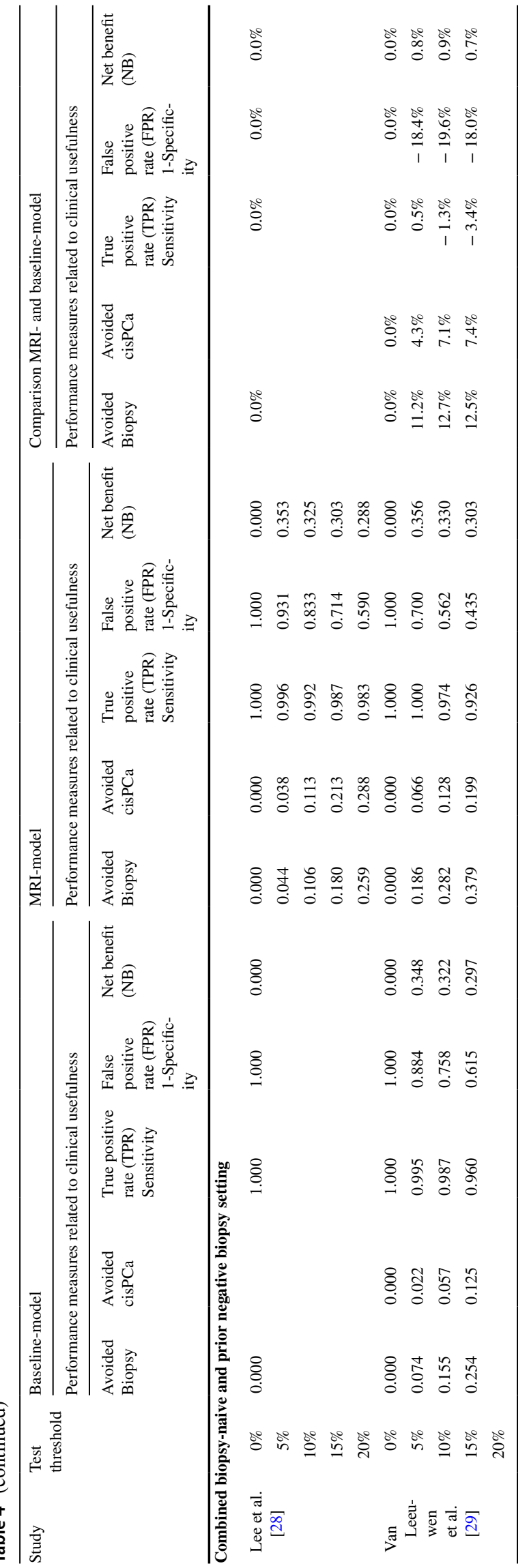

also be accurately assessed, and avoiding the need for performing a TRUS [33, 34]. It provides information on probably the most strong predictor for biopsy outcome currently available, i.e., PSA-density. Therefore, the results of the MRI-based models can be used to counsel men in biopsy decision-making, even before planning a TRUS. Still, the indication for prostate MRI should be critically evaluated, when even unnecessary mpMRI's can be avoided by multivariate risk prediction $[35,36]$. In such a scenario, at first the mpMRI could be circumvented using DRE to the roughly estimate prostate volume. Subsequently, mpMRI could only be utilized in men that most likely have an elevated PSA not related to BPH [37-41].

Despite the good performance of these constructed and published MRI risk prediction models, in combination with the potential advantages, we need to address some limitations for its generalizability and practicality in daily clinic.

The accuracy of the systematic biopsy is dependent on the number of cores. Most studies reported a transrectal approach with a median of 12 biopsy cores (Table 1) [20, 22-27, 30]. However, three studies used a transperineal approach with biopsy cores ranging from 24 to 40 [21, $28,29]$. This is important to realize when such a model is applied in a setting where this biopsy technique is not implemented as a routine daily based practice. Furthermore, the results of biopsy schemes involving saturation biopsies appear to have a higher concordance rate with results from prostatectomy than a scheme involving 12 cores, indicating that these tools predicts more accurate the risk of csPCa [42]. However, in a literature review the increase in diagnostic yield becomes marginal as the number of cores increases above 12 [42, 43]. Furthermore, some existing risk calculators are derived from a six or eight core approach, in which the reference test may be less reliable [44, 45]. Moreover, different targeting methods (MRI/US fusion or cognitive biopsy) were used. Subsequently, when systematic biopsies are performed with the knowledge of the mpMRI findings, the results from this input variable might overestimate the diagnostic performances of the multivariable model.

Next to prevalence of csPCa, the characteristics of the population tested, may influence the outcome of the models. Therefore, again, the applicability of the predicted risk should match with the risk in the investigated population. For example, higher PSA value combined with a higher positive rate of DRE or abnormal TRUS findings, may select a population with a high-risk for $\mathrm{csPCa}$, and may reduce the additional value of mpMRI. Moreover, when experienced radiologists have interpreted the mpMRIs in the cohorts used for the predictive models, the results of these models may not be applicable to less experienced radiologists.

The constructed MRI risk prediction models were based on retrospective data with a probable risk of selection bias. Furthermore, these models were constructed with relatively 
small sample sizes, mostly from a single institution that may hamper the extrapolation and interpretation into larger cohorts.

Internal validation was performed on an even smaller (single-center) sample size and AUCs dropped in some examples [24, 25, 29]. Prediction tools require external validation in a multicenter study to assess their wider applicability. Only one study externally validated a constructed MRI risk model [30] with a small drop in AUC as compared to the development cohort [26].

Novel biomarkers for PCa with prognostic value will be developed. Ancillary tests such as the prostate cancer antigen 3 (PCA3) test, prostate health index, 4Kscore test, and ConfirmMDx may also be considered to provide further reassurance about omitting prostate biopsy. As a next step, these markers will be integrated in the multivariate risk prediction tools. One study reported the analysis of PCA3 in their report; however, in the multivariate analysis PCA3 did not sustain significance as an independent predictor [24].

Above all, we should take care investigating these MRIbased risk prediction models and make sure that in our surge of enthusiasm we do not throw the baby out with the bathwater. We do have already validated risk prediction models with satisfactory performance [13-19], however, the utility of these models are not completely adopted in daily practice, despite recommendation by international guidelines [3, 4]. Further improvement in selecting only those men who will benefit from mpMRI will be essential in the near future. The additional value of mpMRI in risk prediction on top off current models should be carefully analyzed. These new MRI risk assessment tools require external validation on different patient populations who possess varying baseline risks to ensure that the risk prediction tool performs satisfactorily prior to implementation in clinical practice.

\section{Conclusions}

Due to the predictive value of mpMRI in prostate cancer diagnosis, recently new multivariate risk prediction tools have been constructed, with the inclusion of the mpMRI suspicion score. All MRI risk prediction models had a high accuracy with area's under the receiver-operating characteristic curves ranging from 0.78 to 0.93 , and suggest that the usage of these tools would allow the selective identification of patients with significant disease. By analyzing individual performance parameters, physician and patient may be better informed to decide whether or not to undergo further biopsy testing.
Author contributions IGS project development, data collection or management, data analysis and interpretation, manuscript writing. Final approval of manuscript done by all authors.

\section{Compliance with ethical standards}

Conflict of interest The authors have no conflicts of interest to declare.

Ethical approval Research did not involve human participants and/or animals.

Informed consent No informed consent.

Open Access This article is distributed under the terms of the Creative Commons Attribution 4.0 International License (http://creativeco mmons.org/licenses/by/4.0/), which permits unrestricted use, distribution, and reproduction in any medium, provided you give appropriate credit to the original author(s) and the source, provide a link to the Creative Commons license, and indicate if changes were made.

\section{References}

1. Borghesi M, Ahmed H, Nam R, Schaeffer E, Schiavina R, Taneja $S$ et al (2017) Complications after systematic, random, and imageguided prostate biopsy. Eur Urol 71:353-365

2. Schroder FH, Hugosson J, Roobol MJ, Tammela TL, Zappa M, Nelen V et al (2014) Screening and prostate cancer mortality: results of the European Randomised Study of Screening for Prostate Cancer (ERSPC) at 13 years of follow-up. Lancet 384:2027-2035

3. European Association of Urology (EAU) (2018) Guidelines on prostate cancer. http://uroweb.org/guideline/prostate-cancer/

4. National Complrehensive Cancer Network (NCCN) (2018) Guidelines on Prostate Cancer: 2018 update. https://www.nccn.org/profe ssionals/physician_gls/default.aspx

5. Boesen L, Nørgaard N, Løgager V et al (2018) Assessment of the diagnostic accuracy of biparametric magnetic resonance imaging for prostate cancer in biopsy-naive men: the biparametric mri for detection of prostate cancer (bidoc) study. JAMA Netw Open 1:e180219

6. Rouviere O, Puech P, Renard-Penna R, Claudon M, Roy C, Mège-Lechevallier F, et al (2019) Diagnostic added value of prostate systematic and targeted biopsy based on multiparametric MRI in biopsy-naïve patients: the MRI-FIRST study. Lancet Oncol 20(1):100-109. doi: https://doi.org/10.1016/S1470 $-2045(18) 30569-2$

7. van der Leest M, Cornel E, Israel B, Hendriks R, Padhani AR, Hoogenboom M, et al (2018) Head-to-head comparison of transrectal ultrasound-guided prostate biopsy versus multiparametric prostate resonance imaging with subsequent magnetic resonanceguided biopsy in biopsy-naive men with elevated prostate-specific antigen: a large prospective multicenter clinical study. Eur Urol. pii: S0302-2838(18)30880-7. https://doi.org/10.1016/j.eurur o.2018.11.023

8. Ahmed HU, El-Shater Bosaily A, Brown LC, Gabe R, Kaplan R, Parmar MK et al (2017) Diagnostic accuracy of multi-parametric MRI and TRUS biopsy in prostate cancer (PROMIS): a paired validating confirmatory study. Lancet 389:815-822

9. Kasivisvanathan V, Rannikko AS, Borghi M, Panebianco V, Mynderse LA, Vaarala MH et al (2018) MRI-targeted or standard biopsy for prostate-cancer diagnosis. N Engl J Med 378(19):17671777. https://doi.org/10.1056/NEJMoa1801993 
10. Panebianco V, Barchetti G, Simone G, Del Monte M, Ciardi A, Grompone MD et al (2018) Negative multiparametric magnetic resonance imaging for prostate cancer: what's next? Eur Urol 74:48-54

11. Moldovan PC, Van den Broeck T, Sylvester R, Marconi L, Bellmunt J, van den Bergh RCN et al (2017) What is the negative predictive value of multiparametric magnetic resonance imaging in excluding prostate cancer at biopsy? A systematic review and meta-analysis from the European Association of Urology Prostate Cancer Guidelines Panel. Eur Urol 72:250-266

12. Padhani AR, Weinreb J, Rosenkrantz AB, Villeirs G, Turkbey B, Barentsz J (2019) Prostate imaging-reporting and data system steering committee: PI-RADS v2 status update and future directions. Eur Urol 75(3):385-396. https://doi.org/10.1016/j.eurur o.2018.05.035

13. Roobol MJ, Steyerberg EW, Kranse R, Wolters T, van den Bergh $\mathrm{RC}$, Bangma $\mathrm{CH}$ et al (2010) A risk-based strategy improves prostate-specific antigen-driven detection of prostate cancer. Eur Urol 57:79-85

14. Ankerst DP, Hoefler J, Bock S, Goodman PJ, Vickers A, Hernandez J et al (2014) Prostate cancer prevention trial risk calculator 2.0 for the prediction of low- vs high-grade prostate cancer. Urology 83:1362-1367

15. Nam RK, Toi A, Klotz LH, Trachtenberg J, Jewett MA, Appu S et al (2007) Assessing individual risk for prostate cancer. J Clin Oncol 25:3582-3588

16. Stephan C, Cammann H, Semjonow A, Diamandis EP, Wymenga LF, Lein M et al (2002) Multicenter evaluation of an artificial neural network to increase the prostate cancer detection rate and reduce unnecessary biopsies. Clin Chem 48:1279-1287

17. Finne $P$, Finne R, Bangma C, Hugosson J, Hakama M, Auvinen A et al (2004) Algorithms based on prostate-specific antigen (PSA), free PSA, digital rectal examination and prostate volume reduce false-positive PSA results in prostate cancer screening. Int J Cancer 111:310-315

18. Karakiewicz PI, Benayoun S, Kattan MW, Perrotte P, Valiquette L, Scardino PT et al (2005) Development and validation of a nomogram predicting the outcome of prostate biopsy based on patient age, digital rectal examination and serum prostate specific antigen. J Urol 173:1930-1934

19. Chun FK, Steuber T, Erbersdobler A, Currlin E, Walz J, Schlomm $\mathrm{T}$ et al (2006) Development and internal validation of a nomogram predicting the probability of prostate cancer Gleason sum upgrading between biopsy and radical prostatectomy pathology. Eur Urol 49:820-826

20. Alberts AR, Roobol MJ, Verbeek JF, Schoots IG, Chiu PK, Osses DF, et al. (2018) Multivariable risk-based patient selection for prostate biopsy after magnetic resonance imaging: improving the European randomized study of screening for prostate cancer risk calculators by combining clinical parameters with the prostate imaging reporting and data system (PI-RADS) score. Eur Urol

21. Radtke JP, Wiesenfarth M, Kesch C, Freitag MT, Alt CD, Celik $\mathrm{K}$ et al (2017) combined clinical parameters and multiparametric magnetic resonance imaging for advanced risk modeling of prostate cancer-patient-tailored risk stratification can reduce unnecessary biopsies. Eur Urol 72:888-896

22. Mehralivand S, Shih JH, Rais-Bahrami S, Oto A, Bednarova S, Nix JW et al (2018) A magnetic resonance imaging-based prediction model for prostate biopsy risk stratification. JAMA Oncol 4:678-685

23. Fang D, Zhao C, Ren D, Yu W, Wang R, Wang H et al (2016) Could magnetic resonance imaging help to identify the presence of prostate cancer before initial biopsy? The development of nomogram predicting the outcomes of prostate biopsy in the Chinese population. Ann Surg Oncol 23:4284-4292
24. Bjurlin MA, Rosenkrantz AB, Sarkar S, Lepor H, Huang WC, Huang R et al (2018) Prediction of prostate cancer risk among men undergoing combined MRI-targeted and systematic biopsy using novel pre-biopsy nomograms that incorporate MRI findings. Urology 112:112-120

25. Niu XK, Li J, Das SK, Xiong Y, Yang CB, Peng T (2017) Developing a nomogram based on multiparametric magnetic resonance imaging for forecasting high-grade prostate cancer to reduce unnecessary biopsies within the prostate-specific antigen gray zone. BMC Med Imaging 17:11

26. Truong M, Wang B, Gordetsky JB, Nix JW, Frye TP, Messing EM et al (2018) Multi-institutional nomogram predicting benign prostate pathology on magnetic resonance/ultrasound fusion biopsy in men with a prior negative 12-core systematic biopsy. Cancer 124:278-285

27. Huang C, Song G, Wang H, Ji G, Li J, Chen Y et al (2018) MultiParametric magnetic resonance imaging-based nomogram for predicting prostate cancer and clinically significant prostate cancer in men undergoing repeat prostate biopsy. Biomed Res Int 2018:6368309

28. Lee SM, Liyanage SH, Wulaningsih W, Wolfe K, Carr T, Younis C et al (2017) Toward an MRI-based nomogram for the prediction of transperineal prostate biopsy outcome: a physician and patient decision tool. Urol Oncol 35:664.e11-664.e18

29. van Leeuwen PJ, Hayen A, Thompson JE, Moses D, Shnier R, Bohm $\mathrm{M}$ et al (2017) A multiparametric magnetic resonance imaging-based risk model to determine the risk of significant prostate cancer prior to biopsy. BJU Int 120:774-781

30. Bjurlin MA, Renson A, Rais-Bahrami S, Truong M, Rosenkrantz AB, Huang R, et al. (2018) Predicting benign prostate pathology on magnetic resonance imaging/ultrasound fusion biopsy in men with a prior negative 12-core systematic biopsy: external validation of a prognostic nomogram. Eur Urol Focus

31. Steyerberg EW, Vickers AJ, Cook NR, Gerds T, Gonen M, Obuchowski $\mathrm{N}$ et al (2010) Assessing the performance of prediction models: a framework for traditional and novel measures. Epidemiology 21:128-138

32. Gayet M, Mannaerts CK, Nieboer D, Beerlage HP, Wijkstra H, Mulders PFA et al (2018) Prediction of prostate cancer: external validation of the ERSPC risk calculator in a contemporary dutch clinical cohort. Eur Urol Focus 4:228-234

33. Haas M, Gunzel K, Miller K, Hamm B, Cash H, Asbach P (2017) Is the ellipsoid formula the new standard for 3-tesla MRI prostate volume calculation without endorectal coil? Urol Int 98:49-53

34. Bezinque A, Moriarity A, Farrell C, Peabody H, Noyes SL, Lane BR (2018) Determination of prostate volume: a comparison of contemporary methods. Acad Radiol 25:1582-1587

35. Mannaerts CK, Gayet M, Verbeek JF, Engelbrecht MRW, SavciHeijink CD, Jager GJ et al (2018) Prostate cancer risk assessment in biopsy-naïve patients: the rotterdam prostate cancer risk calculator in multiparametric magnetic resonance imaging-transrectal ultrasound (TRUS) fusion biopsy and systematic TRUS biopsy. Eur Urol Oncol 1:109-117

36. Alberts AR, Schoots IG, Bokhorst LP, van Leenders GJ, Bangma $\mathrm{CH}$, Roobol MJ (2016) Risk-based patient selection for magnetic resonance imaging-targeted prostate biopsy after negative transrectal ultrasound-guided random biopsy avoids unnecessary magnetic resonance imaging scans. Eur Urol 69:1129-1134

37. Hansen NL, Barrett T, Koo B, Doble A, Gnanapragasam V, Warren A et al (2017) The influence of prostate-specific antigen density on positive and negative predictive values of multiparametric magnetic resonance imaging to detect Gleason score 7-10 prostate cancer in a repeat biopsy setting. BJU Int 119:724-730

38. Hansen NL, Barrett T, Kesch C, Pepdjonovic L, Bonekamp D, O'Sullivan R et al (2018) Multicentre evaluation of magnetic resonance imaging supported transperineal prostate biopsy in 
biopsy-naive men with suspicion of prostate cancer. BJU Int 122:40-49

39. Distler FA, Radtke JP, Bonekamp D, Kesch C, Schlemmer HP, Wieczorek K et al (2017) The value of PSA density in combination with PI-RADS for the accuracy of prostate cancer prediction. J Urol 198:575-582

40. Washino S, Okochi T, Saito K, Konishi T, Hirai M, Kobayashi $\mathrm{Y}$ et al (2017) Combination of prostate imaging reporting and data system (PI-RADS) score and prostate-specific antigen (PSA) density predicts biopsy outcome in prostate biopsy naive patients. BJU Int 119:225-233

41. Felker ER, Raman SS, Margolis DJ, Lu DSK, Shaheen N, Natarajan $S$ et al (2017) Risk stratification among men with prostate imaging reporting and data system version 2 category 3 transition zone lesions: is biopsy always necessary? AJR Am J Roentgenol 209:1272-1277

42. Bjurlin MA, Carter HB, Schellhammer P, Cookson MS, Gomella LG, Troyer D et al (2013) Optimization of initial prostate biopsy in clinical practice: sampling, labeling and specimen processing. J Urol 189:2039-2046

43. Ouzaid I, Xylinas E, Campeggi A, Hoznek A, Vordos D, Abbou CC et al (2013) Contemporary pathologic characteristics and oncologic outcomes of prostate cancers missed by 6- and 12-core biopsy and diagnosed with a 21-core biopsy protocol. World J Urol 31:869-874

44. Kranse R, Roobol M, Schroder FH (2008) A graphical device to represent the outcomes of a logistic regression analysis. Prostate 68:1674-1680

45. Thompson IM, Ankerst DP, Chi C, Goodman PJ, Tangen CM, Lucia MS et al (2006) Assessing prostate cancer risk: results from the prostate cancer prevention trial. J Natl Cancer Inst 98:529-534

Publisher's Note Springer Nature remains neutral with regard to jurisdictional claims in published maps and institutional affiliations. 